\title{
Perfil clínico, epidemiológico e laboratorial da tuberculose entre 2014 a 2019 no estado
} da Bahia

Clinical profile, epidemiologic and laboratory of tuberculosis between 2014 to 2019 in the state of the Bahia Perfil clínico, epidemiológico y de laboratorio de la tuberculosis entre 2014 y 2019 en el estado de Bahia

\begin{abstract}
RESUMO
Objetivo: Analisar as características clínicas-epidemiológicas dos casos de tuberculose com confirmações laboratoriais, evidenciando a distribuição da doença na Bahia entre 2014 a 2019. Método: Para obtenção dos dados utilizou-se o Sistema de Informação de Agravos e Notificações (SINAN) disponivel para consulta no banco de dados Departamento de Informática do Sistema Único de Saúde (DATASUS), além da utilização de boletins epidemiológicos, websites e artigos científicos indexados nas bases de dados. Resultados: Constatou-se um aumento no coeficiente de incidência da tuberculose nos três últimos anos, no estado da Bahia. A caracterização dos portadores de tuberculose nessa região trata-se de uma população com faixa etária de 20 a 39 anos, predominância pela forma pulmonar e destaca que, quanto aos recursos para diagnóstico laboratorial da tuberculose, a baciloscopia do escarro demostrou maior representatividade. Conclusão: Espera-se que os resultados deste estudo fortaleçam a assistência em saúde, aproximando o paciente das medidas de controle da tuberculose.
\end{abstract}

DESCRITORES: Epidemiologia; Mycobacterium tuberculosis; Tuberculose Pulmonar.

\section{ABSTRACT}

Objective: To analyze the clinical-epidemiological characteristics of tuberculosis cases with laboratory confirmation, showing the distribution of the disease in Bahia between 2014 to 2019. Method: To obtain the data, the Diseases and Notifications Information System (SINAN) was used to consultation in the database of the Department of Informatics of the Unified Health System (DATASUS), in addition to the use of epidemiological bulletins, websites and scientific articles indexed in the databases. Results: An increase in the incidence coefficient of tuberculosis was found in the last three years, in the state of Bahia. The characterization of tuberculosis patients in this region is a population aged 20 to 39 years, predominantly due to the pulmonary form and highlights that, regarding resources for laboratory diagnosis of tuberculosis, sputum smear microscopy showed greater representativeness. Conclusion: The results of this study are expected to strengthen health care, bringing the patient closer to tuberculosis control measures.

DESCRIPTORS: Epidemiology; Mycobacterium tuberculosis; Pulmonary Tuberculosis.

\section{RESUMEN}

Objetivo: Analizar las características clínico-epidemiológicas de los casos de tuberculosis con confirmación de laboratorio, mostrando la distribución de la enfermedad en Bahía entre 2014 y 2019. Método: Para obtener los datos, se utilizó el Sistema de Información de Enfermedades y Notificaciones (SINAN) para asistencia en la base de datos del Departamento de Informática del Sistema Único de Salud (DATASUS), además del uso de boletines epidemiológicos, websites y artículos científicos indexados en las bases de datos. Resultados: Hubo un aumento en el coeficiente de incidencia de tuberculosis en los últimos tres años, en el estado de Bahía. La caracterización de los pacientes tuberculosos en esta región es una población de 20 a 39 años, predominantemente por la forma pulmonar y destaca que, en relación a los recursos para el diagnóstico de laboratorio de la tuberculosis, la baciloscopia de esputo fue más representativa. Conclusión: Se espera que los resultados de este estudio fortalezcan la atención de salud, acercando al paciente a las medidas de control de la tuberculosis.

DESCRIPTORES: Epidemiología; Mycobacterium tuberculosis; Tuberculosis pulmonar.

RECEBIDO EM: 16/08/2020 APROVADO EM: 28/08/2020 


\section{Lorena Cristina dos Santos Marques}

Enfermeira. Especialista em Saúde Pública e Discente do Curso de Biomedicina da Faculdade de Tecnologia de Valença. ORCID: 0000-0001-8929-3716

\section{Ohana Luiza Santos de Oliveira}

Mestra em Genética e Biologia Molecular. Especialista de Gestão em Saúde. Biomédica e Docente da Faculdade de Tecnologia de Valença.

ORCID: 0000-0003-3414-6537

\section{Marilane Andrade Pereira}

Mestra em Sistemas Aquáticos Tropicais. Bióloga e Docente da Faculdade de Tecnologia de Valença.

ORCID: 0000-0001-8333-410X

\section{INTRODUÇÃO}

A tualmente, a tuberculose continua apresentando relevância na saúde pública por tratar-se de uma doença infectocontagiosa, cuja ocorrência predomina nos países em desenvolvimento e em condições de pobreza, devido à falta de prevenção e cuidados ${ }^{1}$. A Organização Mundial da Saúde ${ }^{2}$ afirma que dentre as doenças infecciosas que acometem o homem, a tuberculose é mundialmente a mais mortal. Cerca de 4.500 pessoas morrem por dia em decorrência dessa enfermidade, neste mesmo período aproximadamente 30.000 pessoas são infectadas por esta doença que pode ser prevenível e curável ${ }^{2}$.

O Brasil é um dos poucos países com alto número de pessoas portadoras de tuberculose e que recebeu o reconhecimento da OMS por possuir um bom desempenho nos indicadores de incidência, cujas notificações são de quase $90 \%$ de todos os casos no território brasileiro. Essa realidade caracteriza-se em decorrência das ações de controle existentes no país, as quais viabilizam um eficaz monitoramento e controle da situação epidemiológica da doença ${ }^{3}$.

O Ministério da Saúde (MS) desenvolveu estratégias através do Plano Nacional pelo Fim da Tuberculose, cuja execução foi dividida em quatro fases: 2017 a 2020, 2021 a 2025, 2026 a 2030 e 2031 a 2035. Essas etapas visam erradicar a doença, no que tange a questão de prioridade entre as políticas governamentais de Saúde Pública até o ano de 2035. Contudo, algumas barreiras como
O Brasil é um dos poucos países com alto número de

pessoas portadoras de tuberculose

e que recebeu o reconhecimento da OMS por possuir um bom desempenho nos indicadores de incidência, a implantação de novas tecnologias de diagnóstico e tratamento devem ser enfrentadas a fim de alcançar a consolidação das estratégias ${ }^{4}$.

Assim sendo, com o propósito de contribuir com as estratégias de saúde pública, através da identificação de grupos de risco, o presente estudo objetivou analisar as características clínicas-epidemiológicas dos casos de tuberculose com confirmações laboratoriais, evidenciando a distribuição da doença, conforme ciclos anuais que foram devidamente notificados na plataforma do Sistema de Informação de Agravos de Notificação (SINAN) no estado da Bahia entre os anos de 2014 a 2019.

\section{MÉTODO}

Trata-se de uma revisão sistemática da literatura com abordagem descritiva da caracterização da tuberculose, sendo aplicado o método PRISMA para seleção e síntese do estudo 5 . A população de análise foi selecionada a partir dos casos de tuberculose notificados por meio da confirmação laboratorial, no estado da Bahia, no período de 2014 a 2019, baseado em dados secundário, disponibilizados pelo Sistema de Informação de Agravos e Notificações (SINAN).

Dispensou-se a submissão ao Comitê de Ética em Pesquisa com Seres Humanos (CEP), por se tratar de um estudo com utilização de dados secundários disponibilizados gratuitamente e através da plataforma online do Departamento de Informática do Sistema Único de Saúde (DATASUS) via Informações 
de Saúde (TABNET). Os dados para a composição do perfil clínico-epidemiológico foram coletados através das variáveis constantes na ficha de notificação faixa etária, as formas da doença, tipos de admissão, situação de encerramento e diagnóstico laboratorial. As análises dos dados foram realizadas a partir da estatística descritiva básica utilizando o software Microsoft Excel', sendo os dados agrupados em tabelas e em gráficos para facilitar a compreensão e a visualização. de tuberculose, sendo elas: sexo, etnia,

O levantamento dos artigos foi realizado em bases de dados como Literatura Latino-Americana e do Caribe em Ciências da Saúde (LILACS), Scientific Electronic Library Online (SciELO), Ministério da Saúde (MS) e Medical Literature Analysis and Retrieval System Online (MEDLINE), os descritores utilizados foram Epidemiologia, Mycobacterium tuberculosis e tuberculose pulmonar foram obtidos a partir da terminologia em saúde consultada nos Descritores em Ciências da Saúde (DeCS).

Figura 1. Número de casos e coeficiente de incidência da tuberculose (por 100.000 habitantes), no período de 2014 a 2019, no estado da Bahia.

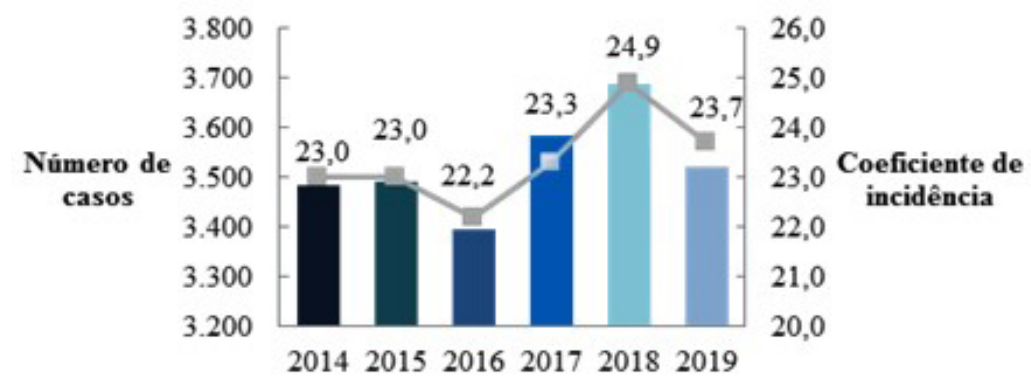

Número de casos

- Coeficiente de incidência por 100.00 hab

Fonte: Dados extraídos do SINAN, 2020.

Tabela 1. Perfil clínico-epidemiológico dos casos de tuberculose, incluindo todas as formas e todos os tipos de admissão da doença, na Bahia de 2014-2019.

$\begin{array}{lllllllll}\text { Variáveis } & 2014 & 2015 & 2016 & 2017 & 2018 & 2019 & \text { Total } & \begin{array}{c}\text { Total } \\ \%\end{array}\end{array}$

Formas Clínicas

Pulmonar

$3.420 \quad 3.375$

3.292

3.456

3.580

3.43730 .560

97

Extrapulmonar

41

55

53

77

64

50

340

2

Pulmonar e

Extrapulmonar

23

60

52

49

45

35

264

1

Tipos de Admissão

$\begin{array}{lcccccccc}\text { Caso novo } & 2.872 & 2.816 & 2.691 & 2.815 & 2.855 & 2.783 & 16.832 & 80 \\ \text { Recidiva } & 235 & 229 & 225 & 210 & 220 & 195 & 1314 & 6,2 \\ \begin{array}{l}\text { Reigresso após } \\ \text { abandono }\end{array} & 194 & 204 & 217 & 275 & 322 & 283 & 1495 & 7,1 \\ \begin{array}{l}\text { Não sabe } \\ \text { Transferência }\end{array} & 10 & 12 & 21 & 16 & 13 & 16 & 88 & 0,4 \\ \text { Pós óbito } & 173 & 226 & 237 & 260 & 272 & 235 & 1403 & 6,6 \\ \text { Fonte: Dados extraídos do SINAN, 2020. } & 0 & 3 & 6 & 6 & 7 & 10 & 32 & 0,2\end{array}$

Foram aplicados os seguintes critérios de inclusão: todos os casos notificados e com diagnóstico laboratorial confirmado de tuberculose na Bahia de 2014 a 2019; além dos artigos científicos que estavam indexados nas bases de dados consultadas, disponíveis em texto completo e gratuito; que se enquadraram na janela temporal dos últimos seis anos; publicações nos idiomas: português e inglês. Foram excluídos da análise, todos os casos de tuberculose que, apesar de notificados, não apresentaram confirmações laboratoriais, além dos artigos disponíveis eletronicamente em que a leitura do título e do resumo não era relevante para o estudo.

\section{RESULTADOS}

No período de 2014 a 2019, foram realizadas 21.164 notificações de casos de tuberculose com confirmações laboratoriais no estado da Bahia. A Figura 1 apresenta os casos de tuberculose registrados a partir da confirmação laboratorial, de acordo com o ano de notificação, bem como os coeficientes de incidência de tuberculose por 100.000 habitantes de 2014 a 2019. Na Bahia, o coeficiente de incidência da tuberculose passou de 23,0/100 mil habitantes em 2014 para 23,7/100 mil habitantes em 2019, representando um aumento de $3 \% \mathrm{em}$ casos novos da doença.

Ao analisar a variável faixa etária, os jovens e adultos entre 20 a 39 anos foram à população mais atingida com a totalidade de 8.734 casos $(41,3 \%)$. No entanto, a menor incidência foi observada na faixa etária de 1 a 4 anos com 12 casos $(0,1 \%)$, nos anos de 2014 a 2019.

A tabela 1 apresenta o perfil clínico-epidemiológico da população do estudo ( $n=21.164)$, constatou-se que a forma pulmonar corresponde a 20.560 casos (97\%), sendo a forma clínica com maior predomínio em todos os anos. Outra variável verificada refere-se ao tipo de admissão, na qual a incidência da tuberculose apresentou-se de forma elevada, $80 \%$ (16.832) das entradas foram 
Tabela 2. Distribuição dos casos de tuberculose, segundo diagnóstico laboratorial, na Bahia, no período de 2014-2019.

$$
\text { Variáveis } \quad \begin{array}{llllllll}
2014 & 2015 & 2016 & 2017 & 2018 & 2019 & \text { Total } & \begin{array}{c}
\text { Total } \\
\%
\end{array}
\end{array}
$$

\section{$1^{\text {a }}$ Baciloscopia do escarro}

Ignorado/ Branco

0

Positivo

3.168

0

0

0

0

0

Negativo

257

194

156

2.887

2.899

2810

17.619

83

Não realizado $59 \quad 297$

238

220

224

158

1.209

6

Não se aplica

0

69

78

117

445

$466 \quad 1.863$

9

\section{$2^{\text {a }}$ Baciloscopia do \\ escarro}

Ignorado/ Branco

1.106

3.489

3.396

3.582

3.869

3.519

18.781

89

Positivo

1.491

0

0

0

0

1

1.494

7

Negativo

688

0

0

0

0

0

199

0

0

0

2

1

\section{Cultura do escarro}

Ignorado/ Branco

Positivo

0

0

0

0

0

0

0

3

Negativo

154

563

689

683

742

508

3.772

0

Em andamento

199

265

224

171

192

115

1.121

18

Não realizado

$2.544 \quad 2.505$

109
2.375

206

192

$512 \quad 13.75$

5

6

Fonte: Dados extraídos do SINAN, 2020.

de casos novos referentes ao acumulativo nos anos de 2014 a 2019.

A tabela 2 sintetiza os dados de notificações no período de 2014 a 2019 com referência a confirmação laboratorial, apontando a elevada positividade para a $1^{\text {a }}$ baciloscopia do escarro totalizando $83 \%$ dos resultados. Nesse mesmo período, fica evidente o alto índice de casos ignorados e/ou em brancos para a $2^{a}$ baciloscopia do escarro, bem como o predomínio no número de casos em que não foi realizada a cultura do escarro.

\section{DISCUSSÃO}

Assim como registrado na Bahia, no Brasil entre 2014 a 2018, o coeficiente de incidência da tuberculose nos anos de 2017 e 2018 se elevou em relação aos anos precedentes, ratificando a necessi- dade em realizar maiores esforços para o controle da doença. Em 2018 o Ministério da Saúde distribuiu mais de 70 aparelhos de Teste Rápido Molecular para Tuberculose, essa ação ampliou a cobertura para 135 cidades que compõem a Rede de Teste Rápido Molecular da Tuberculose (RTR-TB) do Sistema Único de Saúde (SUS) contando, agora, com 249 equipamentos6. Sugere-se que este processo tenha contribuído em relação ao aumento no número de notificações, e por consequência, no coeficiente de incidência da tuberculose no estado da Bahia. Embora, a busca ativa dos casos de tuberculose ainda não seja uma ação prioritária nos serviços de saúde, a ampliação do acesso às ferramentas de diagnóstico é uma estratégia útil no controle da doença ${ }^{7}$.

Estando em conformidade ao preconizado pela OMS, o estado da Bahia re- gistrou uma redução anual no coeficiente de incidência da tuberculose de $4,8 \%$ em 2019 quando comparado ao ano anterior, possivelmente em decorrência da implantação de políticas públicas de saúde voltadas para o controle da tuberculose.

Quanto a faixa etária, a população mais atingida na Bahia ocorreu entre as idade de 20 a 39 anos $(41,3 \%)$ por se tratar de uma das fases mais ativas da vida, semelhante à predominância no estudo realizado por Rodrigues et al.8, que evidenciaram uma frequência maior da doença na mesma faixa etária e Freitas et al. ${ }^{1}$, os quais afirmam que os dados referentes a faixa etária acompanham o padrão nacional, com predomínio da tuberculose em idade produtiva, fase da vida cujos indivíduos encontram-se economicamente ativos, ou seja, executando suas atividades laborais a fim de garantir a subsistência familiar e acabam por protelar a procura por serviços de saúde?.

Entretanto, no presente estudo as crianças com faixa etária entre 1 a 4 anos expressam a doença com menores ocorrências registradas. De acordo com o Ministério da Saúde esse fato pode estar atrelado à dificuldade em diagnosticar e aos sintomas inespecíficos, associados a fatores que inviabilizam a avaliação, podendo elevar o risco de evolução da doença para as formas mais graves e até a morte10. Outra vertente é justamente a maturidade imunológica da criança e a resposta induzida ao fator vacinal, tendo em vista que o Sistema Único de Saúde (SUS) dispõe dentro do quadro vacinal o Bacillus Calmette-Guérin (BCG) cuja eficácia varia em torno $0 \%$ a $80 \%$ viabilizando a redução da incidência de tuberculose nas formas mais graves em crianças vacinadas ${ }^{11}$.

Ao analisar os dados clínico-epidemiológicos, a forma clínica de manifestação da doença mais predominante no estado da Bahia entre 2014 e 2019 foi a tuberculose pulmonar. Essa característica clínica pode ser explicada, pelo fato do bacilo possuir preferência pelos pulmóes, visto que é considerada uma bactéria aeróbica estrita ${ }^{12}$. Resultados similares 
coincidem com o estudo realizado por Cardoso; Pitangueira ${ }^{13}$ e Silva; Silveira; Silva14, os quais descrevem o predomínio da forma pulmonar sobre as demais apresentações clínicas da tuberculose.

Quanto aos casos notificados, conforme tipo de admissão da tuberculose observou-se no estudo que, em todos os anos, o tipo de entrada relacionado aos casos novos prevaleceu sobre os outros tipos, conforme apontam vários estu$\operatorname{dos}^{15}{ }^{16}$. Esse fato justifica-se em razão do déficit de diagnóstico favorecendo para a perpetuação da cadeia de transmissão, bem como, no acompanhamento do portador da doença ${ }^{17}$.

Quando investigadas as variáveis para diagnóstico laboratorial da doença, têm-se a primeira baciloscopia direta do escarro como o método mais predominante e utilizado pela saúde pública, visto que constitui o principal método diagnóstico por ser mais acessível, além de possuir custo inferior aos demais e rápido resultado18. Entretanto, possui limitações quanto à positividade, devido à baixa sensibilidade e especificidade podendo comprometer as interpretações dos exames ${ }^{1}$.

No presente estudo, os achados para a segunda baciloscopia, sugerem que o alto percentual de casos ignorado e/ou em branco não permitiu um diagnóstico por meio de confirmação laboratorial, em razão da ausência de informações de diagnósticos. Assis, Amaral, Mendonça $^{19}$, discorrem sobre a importância do preenchimento dos campos quanto às informações de diagnóstico laboratorial e ressalta que a omissão desses dados inviabiliza a inserção da notificação, afinal não há como identificar se o exame não foi solicitado ao paciente ou se não foi
Os dados apresentados denotam a necessidade de realizar novas pesquisas acerca do tema, a fim de conhecer a realidade da tuberculose em determinada região, bem como a caracterização dos portadores da doença, de forma a identificar

precocemente

possíveis riscose evitar a disseminação da doença além do aumento da mortalidade. realizado, remetendo uma fragilidade do sistema de informação.

Neste estudo, ainda quanto ao método de diagnóstico a cultura do escarro não foi realizada em $70 \%$ dos casos. Silva et al. ${ }^{15}$, justificam que esse fato pode ser em decorrência da demora, da complexidade e do elevado custo quando comparada com a baciloscopia. Fatores semelhantes a esses descritos são enfatizados pelo Ministério da Saúde, o qual afirma que embora seja considerado o diagnóstico laboratorial padrão ouro devido a sua elevada especificidade e sensibilidade, o tempo de detecção do crescimento bacteriano poderá se estender e por consequência retardar o diagnóstico, favorecendo a transmissão da doença. Contudo, esse método de diagnóstico continua sendo relevante, pois aumenta em até $30 \%$ o diagnóstico bacteriológico da doença ${ }^{10}$.

\section{CONCLUSÃO}

Os dados apresentados denotam a necessidade de realizar novas pesquisas acerca do tema, a fim de conhecer a realidade da tuberculose em determinada região, bem como a caracterização dos portadores da doença, de forma a identificar precocemente possíveis riscos e evitar a disseminação da doença além do aumento da mortalidade.

Espera-se que os resultados deste estudo fortaleçam a assistência em saúde, aproximando o paciente das medidas de controle da tuberculose, além de contribuir no aprimoramento e nas implementações de medidas e estratégias em saúde que visem o controle da doença e até mesmo a redução na incidência da tuberculose na região. -

\section{REFERÊNCIAS}

1. Freitas WMTM, Santos CC dos, Silva MM, Rocha GA da. Perfil clínico-epidemiológico de pacientes portadores de tuberculose atendidos em uma unidade municipal de saúde de Belém, Estado do Pará, Brasil. Revista Pan-Amaz Saúde. 2016; 7(2): 45-50. Disponível em:< http://scielo.iec.gov.br/scielo.php?script=sci_arttext\&pid=S2176-62232016000200045\&lng=pt\&nrm=iso Acesso em: 26 de abril de 2020.

2. OMS. Reporte Global da Tuberculose. Geneva: Organização 


\section{REFERÊNCIAS}

mundial de saúde, 2019. Disponível em: <https://www.who. int/campaigns/world-tb-day/world-tb-day-2019>. Acesso em: 23 de março de 2020.

3. BRASIL. Ministério da Saúde. Portal do Governo Brasileiro. Brasília (DF); 2017. Disponível em:<http://saude.gov.br/noticias/agencia-saude/41757-brasil-e-um-dos-paises-de-alta-carga-de-tb-com-melhores-indicadores-relacionados-aincidencia-diz-oms>. Acesso em: 11 de março de 2020.

4. Brasil. Ministério da Saúde. Secretaria de Vigilância em Saúde. Departamento de Vigilância das Doenças Transmissiveis. Brasil Livre da Tuberculose: Plano Nacional pelo Fim da Tuberculose como Problema de Saúde Pública. Brasília: Ministério da Saúde. 2017; p.52. Disponível em:<http://bvsms. saude.gov.br/bvs/publicacoes/brasil_livre_tuberculose_plano_nacional.pdf>. Acesso em: 11 de março de 2020.

5. Shamseer L, Moher D, Clarke M, Ghersi D, Liberati A, Petticrew M, Shekelle P, Stewart LA, the PRISMA-P Group. Preferred Reporting Items for Systematic Review and Meta-Analysis Protocols (PRISMA-P) 2015: elaboration and explanation. BMJ 2015.349:g7647. Disponivel em: < https:// www.bmj.com/content/349/bmj.g7647>. Acesso em: 26 de agosto de 2020.

6. Brasil. Ministério da Saúde. Secretaria de Vigilância em Saúde. Brasil Livre da Tuberculose: evolução dos cenários epidemiológicos e operacionais da doença. Boletim epidemiológico. Brasília: Ministério da Saúde. 2019; 50(9). Disponivel em:<https://www.saude.gov.br/images/pdf/2019/ marco/22/2019-009.pdf>.Acesso em: 11 de março de 2020.

7. Cecilio HPM, Higarashi IH, Marcon SS. Opinião dos profissionais de saúde sobre os serviços de controle da tuberculose. Acta paul. Enferm. 2015; 28(1): 19-25. Disponivel em:<https://www.scielo.br/pdf/ape/v28n1/1982-0194ape-028-001-0019.pdf>. Acesso em 21 de maio de 2020.

8. Rodrigues AL, Hydall ARS, Souza DR de, Borges JL, Oliveira DSA, Pereira, JSF. Características e aspectos epidemiológicos dos casos de tuberculose em um município da Amazônia legal. Revista Multidisciplinar de Estudos Científicos em Saúde. 2019; 4(7): 47-53. ). Disponivel em:<https://www.revistaremecs.com.br/index.php/remecs/article/view/287/pdf >. Acesso em: 25 de maio de 2020.

9. Santos ÁN, Santos MR, Gonçalves LVP. Perfil Epidemiológico Da Tuberculose Em Uma Microrregião Da Bahia (20082018). Revista Brasileira de Saúde Funcional. 2020; 10(1): 29. Disponível em:<http://www.seer-adventista.com.br/ojs3/ index.php/RBSF/article/view/1234/938>. Acesso em: 19 de junho de 2020

10. Brasil. Ministério da Saúde. Secretaria de Vigilância em Saúde. Departamento de Vigilância das Doenças Transmissíveis. Manual de Recomendações para o Controle da Tuberculose no Brasil. $2^{a}$ edição. Brasília: Ministério da Saúde. 2019; p.364. Disponível em:<http://bvsms.saude.gov.br/bvs/ publicacoes/manual_recomendacoes_controle_tuberculose_brasil_2_ed.pdf>. Acesso em: 11 de março de 2020.
11. Saroha M, Faridi MM, Batra P, Kaur I, Dewan DK. Immunogenicity and safety of early vs delayed BCG vaccination in moderately preterm (31-33 weeks) infants. Hum Vaccin Immunother. 2015; 11(12): 2864-2871. . Disponivel em:< https://www.tandfonline.com/doi/full/10.1080/21645515.2 015.1074361 >. Acesso em: 19 de junho de 2020.

12. Silva ILC da, Lima LR, Costa MJM, Campelo V. Perfil epidemiológico da tuberculose no município de Teresina-PI de 2008 a 2012. Revista Interdisciplinar Ciências e Saúde. 2017; 4(1): 36-46. Disponível em:< https://revistas.ufpi.br/index. php/rics/article/view/4561/3895>. Acesso em: 22 de maio de 2020

13. Cardoso RSS, Pitangueira CMFC. Perfil Epidemiológico da Tuberculose no Município de Juazeiro-Ba. Rev. Ref. Saúde- FESGO. 2020; 3(1):58-64. Disponível em:< http:// periodicos.estacio.br/index.php/rrsfesgo/article/viewFile/8082/47966657>.Acesso em: 17 de maio de 2020.

14. Silva PMS; Silveira RGL, Silva LF da. Políticas de informação em saúde: uma análise na incidência de tuberculose no município de Brejo Santo-CE. P2P E INOVAÇÃO. 2019; 6(1): 83-100. Disponivel em:< http://revista.ibict.br/p2p/article/ view/4968/4265>. Acesso em: 22 de maio de 2020.

15. Silva WA, Soares YJA, Sampaio JPS, Chaves TVS. Perfil epidemiológico dos casos notificados de tuberculose no Piauí nos anos de 2010 a 2014. Revista Interdisciplinar. 2017; 10(1): 110-117. Disponivel em: <https://revistainterdisciplinar.uninovafapi.edu.br/index.php/revinter/article/view/1095/0\#: :text=Para\%20tanto\%2C\%20utilizou\%2Dse\%20dados,pulmonar\%20(82\%2C2\%25)>. Acesso em: 22 de maio de 2020.

16. Lima SMA, Silva EMM; Lima MJ de; Jucá AM. Caracterização dos casos de tuberculose notificados em um município prioritário do Brasil, de 2011-2015. Revista Eletrônica Acervo Saúde. 2019; 11(13): 482. Disponivel em:< https://acervomais.com.br/index.php/saude/article/view/482>. Acesso em: 21 de maio de 2020.

17. Fontes GJF, Silva TG da, Sousa JCM de, Feitosa ANA, Silva ML, Bezerra ALD, Assis EV. Perfil epidemiológico da tuberculose no Brasil no período de 2012 a 2016, Revista Brasileira de Educação e Saúde. 2019; 9(1): 19-26. Disponível em:<https://www.gvaa.com.br/revista/index.php/REBES/article/view/6376>. Acesso em: 21 de maio de 2020.

18. Souza AC, Custódio FR, Melo OF. Cenário epidemiológico da tuberculose no município de Sobral (CE) entre os anos de 2013 e 2017. Revista Eletrônica Acervo Saúde. 2019; 23: e445. Disponível em:<https://acervomais.com.br/index.php/ saude/article/view/445>. Acesso em: 21 de maio de 2020.

19. Assis VCA, Amaral MPH do, Mendonça AE de. Análise da qualidade das notificações de dengue informadas no sistema de informação de agravos de notificação, na epidemia de 2010, em uma cidade polo da zona da mata do estado de Minas Gerais. Rev. APS. 2014; 17(4): 429-437. Disponivel:< https:/l periodicos.ufjf.br/index.php/aps/article/view/15336>. Acesso em: 24 de maio de 2020. 